University of San Diego

Digital USD

Spring 5-27-2017

\title{
Physician Orders for Life-Sustaining Treatment (POLST) Utilization in a Skilled Nursing Facility: An Educational Quality Initiative
}

\author{
Ryan Hazley BSN, RN, DNP Student \\ University of San Diego, rnhazley@sandiego.edu \\ Karl Steinberg MD, CMD \\ California State University Institute for Palliative Care, karlsteinberg@mail.com \\ Joseph Burkard DNSc, CRNA \\ University of San Diego, jburkard@sandiego.edu
}

Follow this and additional works at: https://digital.sandiego.edu/dnp

Part of the Geriatric Nursing Commons, Health and Medical Administration Commons, Interprofessional Education Commons, Nursing Administration Commons, and the Palliative Care Commons

\section{Digital USD Citation}

Hazley, Ryan BSN, RN, DNP Student; Steinberg, Karl MD, CMD; and Burkard, Joseph DNSc, CRNA, "Physician Orders for Life-Sustaining Treatment (POLST) Utilization in a Skilled Nursing Facility: An Educational Quality Initiative" (2017). Doctor of Nursing Practice Final Manuscripts. 46.

https://digital.sandiego.edu/dnp/46

This Doctor of Nursing Practice Final Manuscript is brought to you for free and open access by the Theses and Dissertations at Digital USD. It has been accepted for inclusion in Doctor of Nursing Practice Final Manuscripts by an authorized administrator of Digital USD. For more information, please contact digital@sandiego.edu. 


\section{University of San Diego}

Digital USD

Spring 5-27-2017

Physician Orders for Life-Sustaining Treatment (POLST) Utilization in a Skilled Nursing Facility: An Educational Quality Initiative

Ryan Hazley BSN, RN, DNP Student

Karl Steinberg MD, CMD

Joseph Burkard DNSc, CRNA

Follow this and additional works at: http://digital.sandiego.edu/dnp

Part of the Geriatric Nursing Commons, Health and Medical Administration Commons, Interprofessional Education Commons, Nursing Administration Commons, and the Palliative Care Commons 
Physician Orders for Life-Sustaining Treatment (POLST)

Utilization in a Skilled Nursing Facility:

An Educational Quality Initiative

\author{
Ryan N. Hazley, BSN, RN ${ }^{\mathrm{a}^{*}}$ \\ DNP, AGNP, FNP Student \\ Karl Steinberg, MD, CMD, HMDC ${ }^{b}$ \\ Joseph Burkard, DNSc, CRNA ${ }^{\mathrm{a}}$
}

${ }^{a}$ Hahn School of Nursing and Health Science, University of San Diego, 5998 Alcala Park San Diego, CA, USA 92110

${ }^{\mathrm{b}}$ California State University Institute for Palliative Care, 333 S. Twin Oaks Valley Rd, San Marcos, CA, USA 92096

* Corresponding author.

Email address: $\underline{\text { rnhazley@ sandiego.edu }}$

(R.N. Hazley) 


\section{Improving Quality of POLST in a Skilled Nursing Facility}

\section{Introduction}

Advance care planning is an integral aspect of the skilled nursing facilities' (SNF) comprehensive care planning process. Involving residents and family members to continuously communicate and evaluate desires and preferences on a routine basis assures the quality of life at the end of life. ${ }^{1}$ However, despite promotion of advance care planning in the palliative care community for decades, as long as aggressive treatment remains the default in the acute medical care community, there remains a discordance with residents' preferences across transitions of care. ${ }^{2-5}$ The origination of the Physician Order for Life Sustaining Treatment (POLST) realigned these misunderstood end-of-life preferences to focus on patients' goals of care, symptom management, and detailed treatment options. A POLST is a clinical tool that serves as a complementing document to the Advance Health Care Directive. The specialized physician order's effectiveness in advance care planning conversations has been designated as the preferred method of palliative care in nursing homes and skilled nursing facilities $(\mathrm{SNFs})^{6,7}$, ensuring patient

preferences match the medical treatments they receive. ${ }^{2}$ The POLST serves as a facilitator to the end-of-life conversation process, improves advance care planning conversations, and serves as a guide for physicians to order medical treatments in keeping with an individual's preference or best interest. Composed of only a few medical treatmentrelated sections, this bright pink order set offers significant advantages over traditional methods to communicate treatment preferences in the nursing facility settings. ${ }^{2,4}$ Although traditional advance directives can be an important tool to assist those facing the end of life, they are not generally considered actionable medical orders by medical 
personnel such as first responders. It has been found that treatment preferences

documented as medical orders are largely consistent with care provided to nursing facility residents with POLST orders. ${ }^{2,4,8}$

\section{Background}

\section{Problem Identification}

As of May 2016, nearly 10 years after California's pioneering efforts through statewide adoption and novel dissemination strengthened by grassroots coalitions supported and funded by the Coalition for Compassionate Care of California (CCCC) and California HealthCare Foundation, California achieved mature POLST program status, being only one of three states meeting the criteria set forth by the National POLST Paradigm Task Force (http://www.polst.org). Local state coalition growth expanded to 27 community POLST coalitions in California, which have actively advocated for quality POLST program diffusion across the state, designing and promoting the POLST education curriculum now used by many POLST programs around the country. ${ }^{3,9}$

With nearly 100 skilled nursing facilities ${ }^{10}$ located in San Diego County, the focus on quality assurance and performance improvement (QAPI) on continued POLST success is a priority to this southern California local community coalition. The recent passage of Senate Bill 19: POLST e-registry, a secure electronic portal to access POLST documents during transitions in care and in times of urgent need, has given these quality assurance efforts even more of a purpose. These rich and meaningful POLST conversations, now eligible for upload in a centralized repository, need to be accurately and completely documented, qualifying as an actionable order that can be followed by a physician or first responders. 
With many SNF licensed staff members not knowledgeable of appropriate POLST utilization, conversation, or documentation practices, the transition to an electronic registry would not be an effective task to achieve. In a 93-bed San Diego SNF, a staff- and workflow-focused gap analysis for POLST utilization in the advance care planning process and conversation was performed. Recurring themes of various challenges and concerns related to POLST utilization in the SNF were reported by a total of 11 staff members during initial surveys. The POLST not being filled out properly accounted for $54 \%$ of staff's response; $36 \%$ had fears of liability and responsibility surrounding the order, $36 \%$ resident and families not understanding implications of POLST, and $36 \%$ reported the process of engaging in a POLST conversation being a challenge. In this SNF there was a $100 \%$ mandated POLST completion by residents and families during the admission process, many of whom were not at the end-of-life, hence not appropriate for POLST based on the National POLST Paradigm Task Force guidelines, and there was an absence of a written POLST policy or procedure availableeven though model policies and procedures for SNFs are available at the CCCC website.

Although there have been significant recent knowledge gains in palliative care and end-of-life clinical education, there remains a critical need for increased system support, public education, and engagement to improve clinician knowledge and confidence to take part in more frequent and productive clinician-patient conversations. ${ }^{11}$ Therefore, this evidence-based practice project focused on answering the question: Among staff in a skilled nursing facility, does implementing a formal POLST education program compared to current practice improve staff knowledge, comfort level, and documentation of the POLST form? 
The purpose of this evidence-based education program was to identify barriers to quality POLST conversations, to increase skilled nursing facility staff's knowledge and comfort level related to POLST utilization, and to improve POLST documentation practices. Staff who knew more about POLST and its role in improving care at end of life, support the importance of widespread dissemination of POLST educational initiatives at nursing facilities, which is vital to the Paradigm's continued success in California, as was in its success in Oregon. ${ }^{12}$ The findings should be extrapolated to other states where similar POLST Paradigm forms ${ }^{13}$ (e.g., POST, MOST, MOLST, COLST, TPOPP) are utilized.

\section{Methods}

\section{Project design and sample}

This evidence-based project evaluated SNF staff's utilization and knowledge of the POLST program as well as documentation practices, which included both consistency (accuracy) and completeness. The training was mandatory during a scheduled one-hour block for staff development that took place once monthly. Participation in the pre- and post-education questionnaires was voluntary. After receiving exemption from the Institutional Review Board, staff were asked to volunteer to participate in the Doctor of Nursing Practice quality improvement educational initiative and consented by completing the first questionnaire. The inclusion criterion was the selection of the answer "yes" to having direct contact with residents and families with POLST or having assisted in completing a POLST. No identification information was collected on the pre- and post- 
education questionnaires. The project was initiated in February 2016 and completed in June 2016.

\section{Intervention}

The educational format consisted of two one-hour training workshops a month apart using the Coalition for Compassionate Care of California (CCCC) and National POLST Program educational curriculum ${ }^{9,13}$. Prior to each of the two workshops, a questionnaire was completed by staff to assess basic knowledge of POLST as well as concerns and challenges completing the POLST with residents and families. Staff completed their third and final questionnaire in the last month to assess for knowledge retention of the educational material. Monthly chart audits also were performed to assess for improvement in documentation consistency (accuracy) and completeness.

Documentation consistency criteria were defined as the accurate concordance of data in Sections A/B and between the Advance Health Care Directive (if available) and the POLST.

To better ensure the sustainability of this educational quality initiative, two Staff Resource Guides were assembled using up to date 2016 National POLST Paradigm curriculum materials provided publicly by the CCCC on the California POLST websites $^{9,13}$ (http://coalitionccc.org/tools-resources/nursing-homes/, capolst.org) to be utilized for new hire education, and reinforcement of education as needed. A tri-fold poster board displayed in the Staff Development room during the course of the initiative included POLST Education with FAQ's, an 'easy-to-read' synopsis of POLST's importance, differences between POLST and the Advance Directive, and a packet of “Consumer Resource Guides. Staff badge/pocket card holders 4x3” in size were made as 
a quick reference guide for conversation cues and served as a convenient reminder that was handy "on-the-go."

\section{Measures}

Identical 19-item questionnaires were provided to staff a total of 3 times during the initiative. The questionnaires were completed before the first training to assess baseline knowledge, immediately after the second training, and one month later to assess for knowledge retention and reduction in challenges and concerns posing barriers to POLST completion related to improved knowledge. The questions provided were adapted from the CA POLST Knowledge Pre-Survey ${ }^{14}$ and CCCC's QAPI toolkit ${ }^{15}$ that have been used in past research. The knowledge section consisted of seven true/false/do not know questions ranging from interdisciplinary involvement to accurate documentation on the various sections of POLST. An additional yes-or-no question was added to assess for uptake of the POLST Program educational/resource materials and decision making guides during POLST conversations. The six demographic questions included information such as age, title, educational level, preferred learning methods, and training received in POLST utilization.

\section{Data analysis}

The computer program SPSS version 23 was used to analyze the pre- and poststaff questionnaire results in Likert-like scale questions. Mean percent was also done with t-test and ANOVA to assess chart documentation data from yes or no questions.

Descriptive statistics were employed to analyze demographic data.

\section{Results}


Eleven skilled nursing facility staff members participated in the educational initiative, including 5 RNs, 4 LVNs, 1 social services designee, and 1 documentation specialist. A majority of participants were between the ages of 35-44 years old (36.3\%) employed in their selected fields for 5-10 years (45\%), and Associate's degree prepared $(54.5 \%)$.

Staff knowledge significantly improved on average $25 \%$, from $57 \%$ to $82 \%$ (95\% CI, 39, 100) and was statistically significant ( $<<.004$, Table 1). Combined documentation consistency (accuracy) and completeness improved on average 34\%, from $66 \%$ to $100 \%$ (95\% CI, 15,100$)$, and was not statistically significant $(\mathrm{p}=.166$, Table 2 ), although, when combined with the 64\% staff's increased use of POLST educational resources, which supports sustainability of knowledge, it could be considered clinically significant. Post educational initiative, POLST documentation accuracy increased by $34 \%$ in direct relation to the staff's knowledge of accurate documentation, which increased by $37 \%$ overall, but was not statistically significant at $\mathrm{p}=0.069$ (Table 3).

Table 1: Staff POLST Knowledge Scores Pre and Post Education

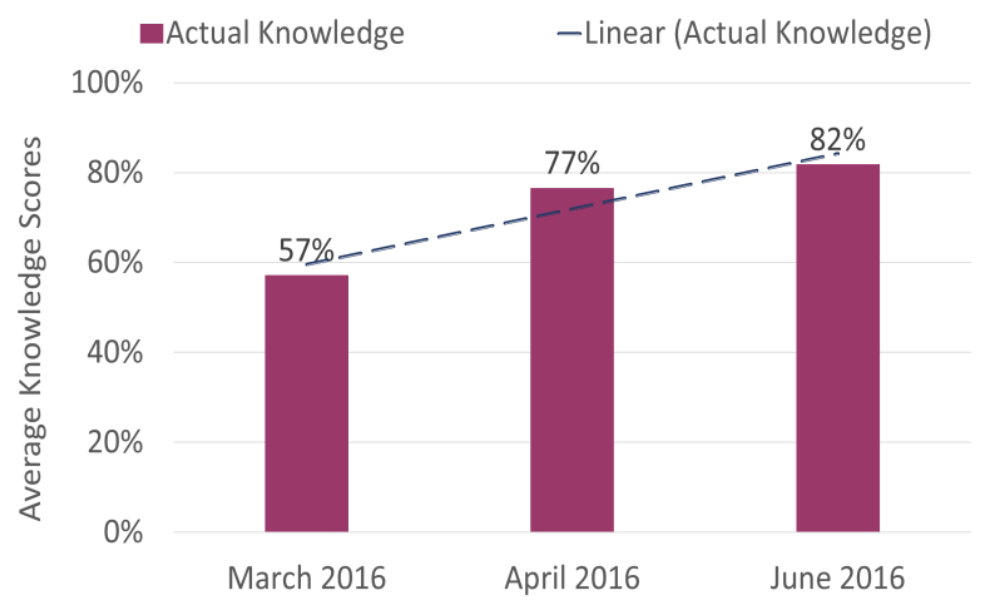


Table 2: Chart Review Comparison of Internal Consistency \& Completeness of POLST Documentation Pre- and Post- Education

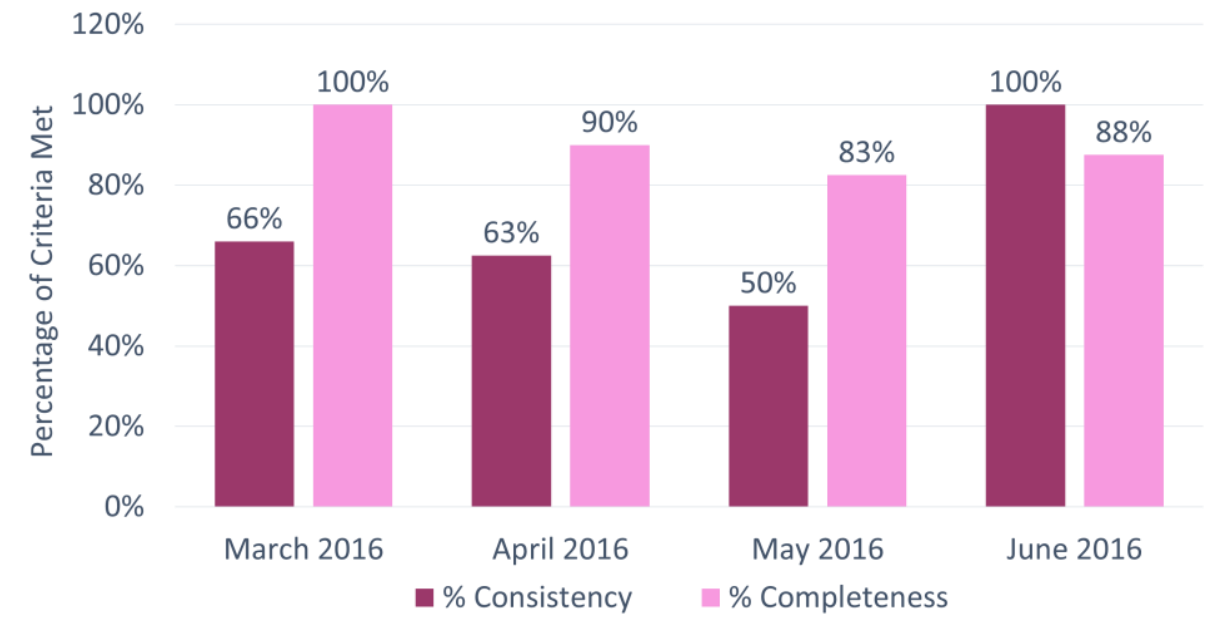

Table 3: POLST Documentation Consistency Scoring vs. Staff Knowledge Scores of Documentation Consistency

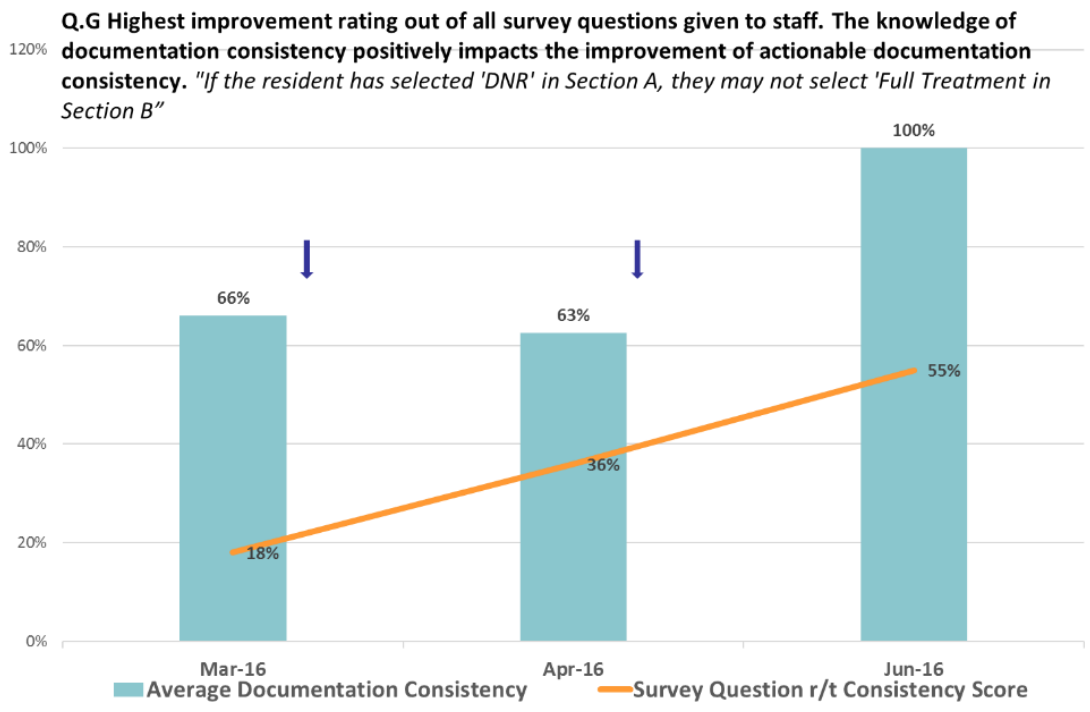


After POLST education, staff knowledge that POLST is voluntary for all residents and by law not mandatory to be completed improved by $64 \%$ As legislation (AB 3000, Statutes 2008, Chapter 266) defines POLST as a physician order to be honored across settings of care, and provides immunity to providers who honor a POLST document in good faith it was noteworthy that staff fears of liability and responsibility with POLST completion decreased by $27 \%$ post education. Family member disagreements remained a concern by $82 \%$ of staff post educational initiative, which posed a barrier to successful POLST completion.

\section{Discussion}

In contrast to a past SNF-focused study ${ }^{12}$ that discussed the increased utilization of POLST being directly related to the increased staff knowledge of POLST, the San Diego SNF's 40-chart audit revealed a 100\% POLST completion rate, and only $27 \%$ of employees believed that they had sufficient POLST training. Of the 40 audited charts, $32.5 \%$ of them had a AHCD accompanying the POLST. With this caveat in mind, and also considering the POLST form is designed for patients in the last year or so of life $\mathrm{e}^{16}$, the emphasis should be placed not on 'POLST forms for everyone,' but on 'Advance Health Care Directives for everyone,' which would legally designate a Durable Power of Attorney. However, despite multiple all-facilities letters (AFLs) from the California Department of Public Health reminding facilities that POLST is never mandatory, the widespread misconception that POLST is required for all nursing home residents persists.

The primary problem found was not a deficit in the paper completion of the POLST form, but rather in the process of the completion of POLST. The required 
involvement of staff and leadership engagement in this culture change initiative was key to its success as compared with the literature, although SNFs had the highest uptake of POLST utilization of any care setting in California; more than one-third of facilities noted several indications for quality improvement, including more staff and leadership engagement ${ }^{3}$. It is evident that most healthcare providers agree that the POLST can improve communication and is useful in initiating conversations about patients' wishes, but challenges that pose barriers to comprehensive POLST conversations focus the direction of future research efforts toward evaluating the gaps in current clinical and professional education as a primary strategy to improve quality ${ }^{2,17}$. Education to help bridge the basic knowledge gaps of SNF personnel around POLST is clearly essential before engagement and development of a robust quality improvement program can occur. It is necessary for frontline staff to gain knowledge of POLST guidelines and standards of practice which includes the understanding that POLST is not another checkoff box form integrated in the SNF admission process.

The inclusion of other licensed healthcare providers to carry out these vital conversations after the passage of Assembly Bill 637, which allows Nurse Practitioners and Physician Assistants to sign POLST forms, creates an interdisciplinary involvement which has been shown to increase quality of coordination of care management, goals of care, and end-of-life conversations ${ }^{18,19,20}$ while also lessening physician burden. This project also created some future research-focused implications such as qualitative assessment of influence and perception of family members on POLST conversations and how this affects end-of-life decision-making experiences.

\section{Limitations}


Due to the smaller facility, the setting, and the need to limit participation to healthcare professionals with current or future direct resident interaction involving POLST conversations, the sample size was small, which could negatively impact the generalizability of the findings. The questionnaire tool's adapted sections, although used in other studies and grant projects, lacks formal validity and reliability testing. Due to ensuring participant anonymity as well as an effort to increase sample size, this project did not collect data that permitted participant matching between pre- and post- survey, but the Director of Staff Development was responsible for keeping a list of the participants to verify involvement. Some staff were not present for all of the face-to-face trainings, but were instructed to review the training reference materials prior to answering the questionnaires. This could have changed understanding and affected scores due to differences in learning styles and teaching modalities. Ongoing barriers and challenges for communication and consistent follow-through were present as the SNF setting has a culture that includes a variety of pressures related in part to being extensively regulated. Barriers were identified related to multiple issues including quality improvement priorities, restructuring, and other day-to-day operational challenges. There was limited information, if any, to validate surrogates' signatures, without Advance Health Care Directive resources available.

\section{Conclusion}

This information emphasizes the critical need for POLST education for nursing home staff. If a higher frequency of POLST forms are being completed but a lesser frequency of training is being conducted to ensure comprehensive and ethically sound conversations are occurring, there needs to be a change in the process and quality of 
POLST utilization. The application of a POLST staff educational initiative in a skilled nursing facility promotes improved patient informed decision-making, enrichment of advance care planning conversations, and overall improvement in quality of life for those with chronic or terminal illness. 


\section{References}

1. Centers for Medicare and Medicaid Services. 42 CFR Parts 405, 410, 411, 414, 425, 495.. Services State Operations Manual. Appendix PP. Available at:

https://www.cms.gov/Regulations-and Guidance/Guidance/Manuals/ Downloads/som107c07; 2015.

2. Hickman, S. E., Keevern, E., \& Hammes, B. J. Use of the physician orders for life-sustaining treatment program in the clinical setting: a systematic review of the literature. J of the Am Geriatrics Society, 2015; 63(2), 341-350.

3. Wenger, N.S., Citko, J., O’Malley, K., Diamant, A., Lorenz, K., Gonzalez, V., Tarn, D.M. Implementation of Physician Orders for Life Sustaining Treatment in nursing homes in California: Evaluation of a novel statewide dissemination mechanism. J of Gen Intern Med. 2012; 28:51-57.

4. Hickman, S.E., Nelson, C.A., Moss, A.H., Tolle, S.W., Perrin, N.A., Hammes, B.J. The consistency between treatments provided to nursing facility residents and orders on the Physician Orders for Life-Sustaining Treatment form. $J$ of American Geriatric Society. 2011; 59:2091-2099.

5. Wilkinson, N. Wenger, and L. R. Shugarman, Literature Review on Advance Directives, U.S. Department of Health and Human Services, Washington, DC, USA, 2007

6. National Quality Forum. A National Framework and Preferred Practices for Palliative Care and Hospice Care Quality: A Consensus Report. Washington, DC: National Quality Forum, 2006; 43-44.

7. Emanuel, L., Glassar Scandrett, K. Decisions at the end of life: have we come of age? BMC Med. 2010:57. Accessed from www.biomedcentral.com/17417015/8/57.

8. Fromme, E. K., Zive, D., Schmidt, T. A., Cook, J. N. B. and Tolle, S. W. Association Between Physician Orders for Life-Sustaining Treatment for Scope of Treatment and In-Hospital Death in Oregon. Journal of the American Geriatrics Society. 2012.

9. Coalition for Compassionate Care of California. Physician Orders for Life Sustaining Treatment. Available at: http://coalitionccc.org/what-we-do/physicianorders-for-life-sustaining-treatment-polst/. http://capolst.org/polst-for-healthcareproviders/. Cited 12.2016.

10. CarePathways. California (CA) Nursing Homes / Skilled Care Facilities by County. Available at: https://www.carepathways.com/nhg-state-CA.cfm. Cited 11.2016.

11. Institute of Medicine. Dying in America: Improving Quality and Honoring Individual Preferences near the End of Life. Key Findings and Recommendations. Academies Press, Washington, D.C. 2014.

12. Vo, H., Pekmezaris, R., Guzik, H., et al. Knowledge and attitudes of health care workers regarding MOLST (Medical Orders for Life-sustaining Treatment) implementation in long-term care facilities. Geriatr Nurs. 2011; 32(1):58-62

13. POLST. About the National POLST Paradigm. National POLST Taskforce. Available at: (http://polst.org/programs-in-your-state/). Cited 10.2016. 
14. McGregor, C. CA POLST Knowledge Pre-Survey. The POLST Quality and Research Toolkit (PQRsT). 9/20/2012. http://polst.org/resources/qualityimprovement/

15. POLST QAPI Toolkit. POLST for HealthCare Providers. http://capolst.org/wpcontent/uploads/2016/06/POLST-QAPI-Toolkit_June2016.pdf. Cited 04.2017.

16. The National POLST Paradigm Program (2015). Available at www.polst.org. Cited 04.03.16.

17. Sabatino, C.P., Karp, N. Improving Advanced Illness Care: The Evolution of State POLST Programs. AARP Public Policy Institute. 2011.

18. Hayes, S., Zive, D., Ferrell, B., Tolle, S.W. The Role of Advanced Practice Registered Nurses in the completion of Physician Orders for Life Sustaining Treatment. J of Palliat Med. 2016; DOI: 10.1089/jpm.2016.0228

19. Hartle, G.A., Thimons, D.G., Angelenni,J. Physician Orders for Life Sustaining Treatment in US Nursing Homes: A case study of CRNP engagement in the care planning process. Nursing Research and Practice. 2014.

20. Waldrop DP, Clemency B, Maguin E et al. Preparation for frontline end-of-life care: Exploring the perspectives of paramedics and emergency medical technicians. J of Palliat Med. 2014;17:338-341. 